\title{
STATISTICAL EVALUATION OF FATIGUE DATA OF COMPONENTS
}

\author{
Chi Nghia Chung*, Zoltan Major \\ Johannes Kepler University Linz, Institute of Polymer Product Engineering, Altenbergerstraße 69, 4040 Linz, \\ Austria \\ * corresponding author: chi_nghia.chung@jku.at
}

Abstract. A variety of steels, cast iron grades and other metals have long been used for the production of machine components. In recent years, however, new materials such as sintered materials and plastics become increasingly important. Because of the large number of different fibers, matrices, stacking sequences, processing conditions and processes and the variety of resulting material configurations it is not possible to rely on proven fatigue models for conventional materials. Moreover, the development of models, which are valid for all composites are generally extremely difficult.

In this work, a possible application of high-performance composites as materials for machine elements are investigated. This study attempts to predict the fatigue behavior and the consequent durability, based on laboratory measurements. Using the statistics program JMP, the aquired data was subjected to a reliability analysis in order to ensure the plausibility, validity and accuracy of the measured values.

KEYWORDS: fatigue models, statistical evaluation, reliability, techniques of parameter fitting, JMP.

\section{INTRODUCTION}

There was a tremendous advance in the field of plastics took in the past few decades. Plastics have become an integral part of our daily life. Polymers are flexible materials which can cover a great range of applications. They replace more and more metal, glass, wood and other materials. The development of novel artificial materials often opens a door to new technologies. Electrical and automotive industry are hard to imagine without plastics, the use of polymer materials has revolutionized medicine.

Cyclically stressed components have a limited durability, therefore it is important to perform fatigue tests or simulations on critical components to predict their lifetime. Figure 1 shows representative loading patterns with constant amplitudes for the whole loading level [1].

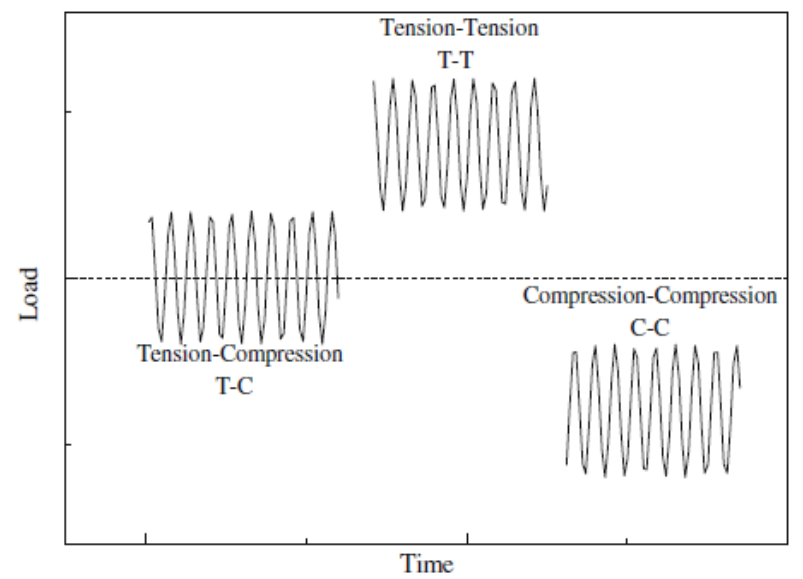

Figure 1. Constant amplitude loading patterns.
Fatigue tests are performed to study the relationship between the fatigue resistance of a material, component or structural element and cyclic loading [2]. It is a slowly progressing damage process. The strengths lie far below the static strength and yield strength.

The objective of this work was to create high cycle fatigue curves from experimental datasets, using suitable material models, similar to the Wöhler curve. The curve should as closely as possible reflect the experimental values. Since specimens and test conditions are never $100 \%$ identical, in two discrete measurements there is always scattering in the results, which can span over a decade in fiber reinforced polymers [1. Therefore to handle and correctly interpret experimental results, statistical methods must be used.

\section{Fatigue Models}

In general, Fatigue models are quantifications of physical material properties. They are independent of the shape of the component and are usually based on experimentally acquired data. The aim of all models is to predict how a component behaves in certain conditions. The relationships are reproduced mathematically afterwards, therefore they are mathematical models.

In this work, the focus will be on presenting fatigue models [3. In order to design a component correctly in terms of fatigue, a complete set of experimentally acquired data is usually required. Since this is not possible for reasons of time and cost, engineers have to rely on predictive models. This models predict the durability N (in cycles) under a given cyclic loading. 


\subsection{The Basquin Model}

The Basquin fatigue model is a linear regression model. In a $\operatorname{logarithmic}$ scale, the durability $(\log \mathrm{N})$ is plotted versus the stress amplitude $(\log \Delta \sigma)$.

$$
\log N=A-B \cdot \log \Delta \sigma, \Delta \sigma \geq \Delta \sigma_{0}, B \geq 0
$$

$\mathrm{A}$ and $\mathrm{B}$ are material parameters that need to be approximated with appropriate fitting methods. In the model, $\Delta \sigma$ is limited by $\Delta \sigma_{0}$, the endurance limit. In this work, the endurance limit is at $40^{6}$ cycles. $\Delta \sigma_{0}$ itself has no direct influence on the model, since it is not considered in the formula.

\subsection{The Strohmeyer Model}

In contrast to the Basquin model the models of Strohmeyer and Weibull are nonlinear. They are shaped by smoothing a piecewise linear function. Again A and B are the material parameters.

$$
\log N=A-B \cdot \log \left(\Delta \sigma-\Delta \sigma_{0}\right), B \geq 0
$$

\subsection{The Weibull Model}

The Weibull model is more complex, since there are more parameters to fit.

$$
\log (N+D)=A-B \cdot \log \left(\frac{\Delta \sigma-\Delta \sigma_{0}}{\Delta \sigma_{s t}-\Delta \sigma_{0}}\right), B \geq 0
$$

$\mathrm{A}, \mathrm{B}$ and $\mathrm{D}$ describe the material parameters and $\Delta \sigma_{s t}$ denotes the ultimate strength.

\subsection{The Bastenaire Model}

$$
\frac{\log N-B}{\Delta \sigma-\Delta \sigma_{0}}=A \cdot \exp \left[-C \cdot\left(\Delta \sigma-\Delta \sigma_{0}\right)\right]
$$

$\mathrm{A}, \mathrm{B}$ and $\mathrm{C}$ express the material parameters. This model will be henceforth denoted as Bastenaire1, because one can find another model of Bastenaire in the literature, which is called Bastenaire2 here. It is quite similar to equation 4 and it is also discussed for the purpose of comparison.

$$
N=A \cdot \exp \left[-C \cdot \frac{\Delta \sigma-\Delta \sigma_{0}}{B}\right] /\left(\Delta \sigma-\Delta \sigma_{0}\right)
$$

\section{Techniques of Parameter FitTING}

In this section, two exemplar fitting methods are discussed in greater detail [4]. Both methods are current and proven estimation methods in statistics.

\subsection{The Method of Least Squares (L2-NorM)}

The method of Least Squares is a mathematical standard procedure for compensation calculation. Here a function is determined, which fits a point cloud as close as possible. A point cloud is a scatter plot, a graphical representation of statistical measurements in a coordinate system.
To illustrate the method, the Basquin model is used as an example. Consider a dependent variable y (in this case, $\log \mathrm{N}$ ), which depends on one or several variables (in this case $\mathrm{A}, \mathrm{B}$ and $\log \Delta \sigma$ ). The relationship between the dependent variable and the arguments are described in a model function $\mathrm{f}$ [7]. The model function $\mathrm{f}$ can be linear, as in this case, but it can also have any other shape (parabolic, exponential, ...). The general form is:

$$
y(x)=f\left(x ; a_{1} \ldots \ldots \ldots a_{m}\right)
$$

In this case:

$$
\log N=A-B \cdot \log \Delta \sigma, \Delta \sigma \geq \Delta \sigma_{0}, B \geq 0
$$

The parameters A and B should be adapted such that bad data points (outliers) have only little effects on the fitting. If no unique solution that perfectly fits the point cloud can be found, then a compromise solution with the smallest overall deviation from the point cloud is the valid criterion. For this purpose, the sum of the squares of the differences between the model function $\mathrm{f}$ and the measured values $\mathrm{y}_{i}$ is formed.

$$
\sum_{i=1}^{n}\left(f\left(x_{i} ; a_{1} \ldots \ldots \ldots a_{m}\right)-y_{i}\right)^{2}
$$

The parameters A and B are adapted until the sum of squares becomes minimal. In this work the fitting procedure carried out with the help of a solver implemented in Excel.

\subsection{The Method of Least Absolute Deviations (L1-Norm)}

The L1 Norm is a more robust fitting method. Outliers are not so strongly weighted. In principle, this method works similarly to the previously explained Method of Least Squares. Instead of the sum of the squares, the absolute sum of the differences between the model function $\mathrm{f}$ and the measured values $\mathrm{y}_{i}$ is calculated.

$$
\text { Abs }\left\|\sum_{i=1}^{n}\left(f\left(x_{i} ; a_{1} \ldots \ldots . . . a_{m}\right)-y_{i}\right)\right\|
$$

Subsequently, the parameters A and B are also be adjusted until the absolute sum is minimized.

\section{Reliability}

The reliability is a measure of the accuracy of the measurement, as well as for the reliability of data. Measurement series with very high reliability are therefore almost free of random errors, which means that they are repeatable at any time under the same measurement conditions and thereby provide approximately the same results [8, 9]. Therefore, one gets a high reliability, when performing controlled and standardized measurements. To examine the reliability, different techniques can be used [10]. Some known techniques are: 
- Test - Retest Reliability

- Parallel - Forms Reliability

- Split - Half Reliability

- Internal - Consistency Reliability.

Reliability analysis can be easily carried out with various computer programs. Known programs are SPSS or, as used in this work, JMP.

\section{Evaluation of Fatigue Data}

Two different materials were tested. Material\#1 is a glass fiber reinforced, semi-crystalline thermoplastic. Material\#2 is a carbon fiber reinforced, semicrystalline thermoplastic, where the bearing was simulated as a compliant bearing. Material\#3 is the same material as Material\#2, however it has been simulated as a rigid bearing.

\subsection{Fatigue Models}

Figure 2 exhibits the applied fatigue models for material\#1. Since for Material\#2 the ultimate strength and the load at the endurance limit could not be investigated, only the Basquin model could be applied 3 In the case of Material\#3 the fitting with the Strohmeyer and the Weibull model resulted in the same curve 4

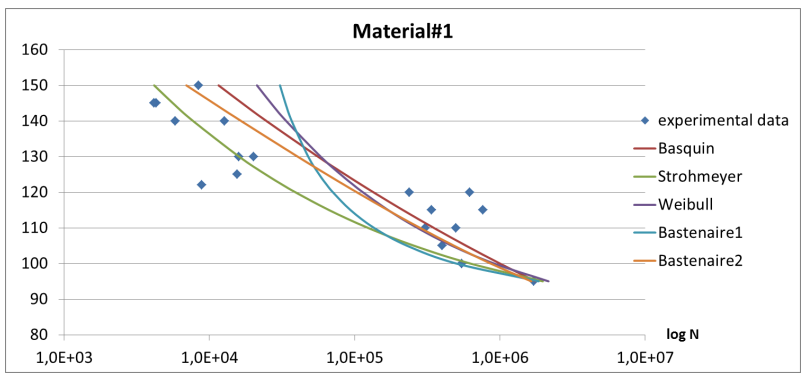

Figure 2. Applied fatigue models for Material\#1.

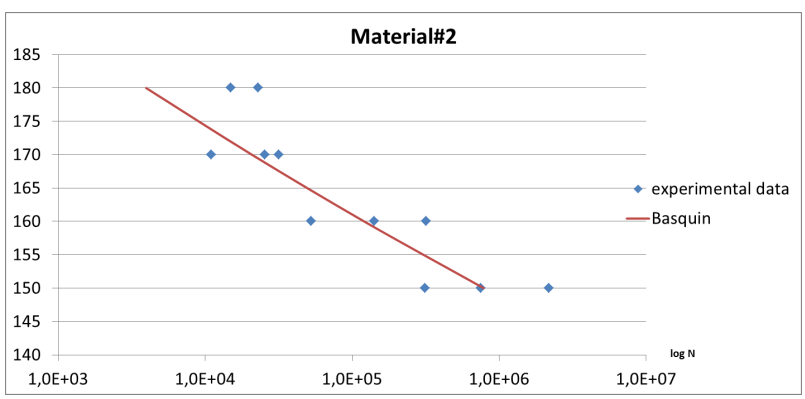

FIgURE 3. Applied fatigue models for Material\#2.

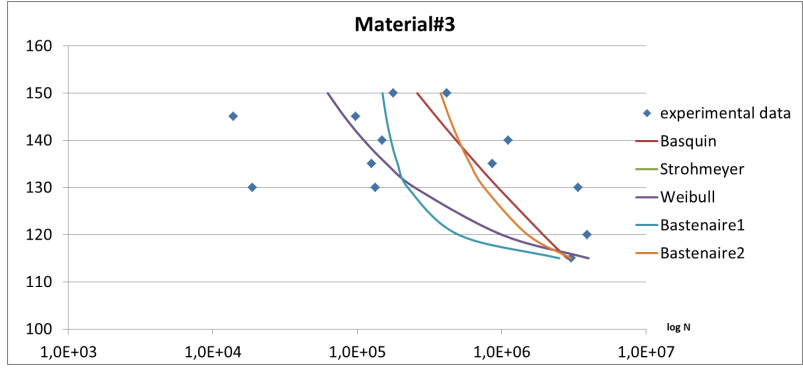

Figure 4. Applied fatigue models for Material\#3.

The software minimized the deviation between the models and the measured values. By comparing the obtained model parameters the optimal model can be easily determined, not only qualitatively but also quantitatively. Figure 5 and 6 show the optimal fatigue models for the materials. Since for Material\#2 only the Basquin model was applied, it was omitted below.

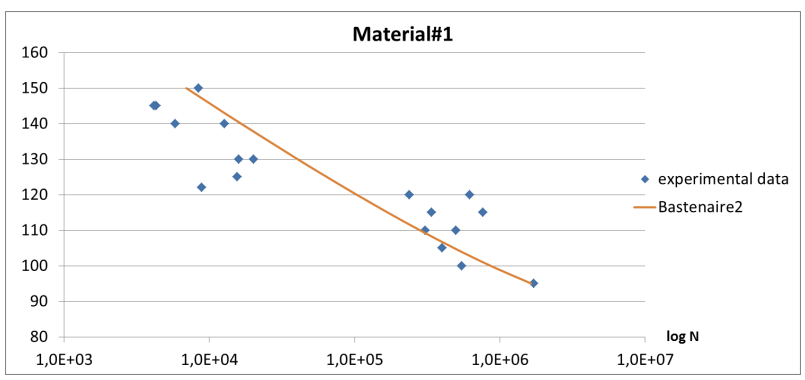

FIGURE 5. Optimal fatigue model for Material\#1.

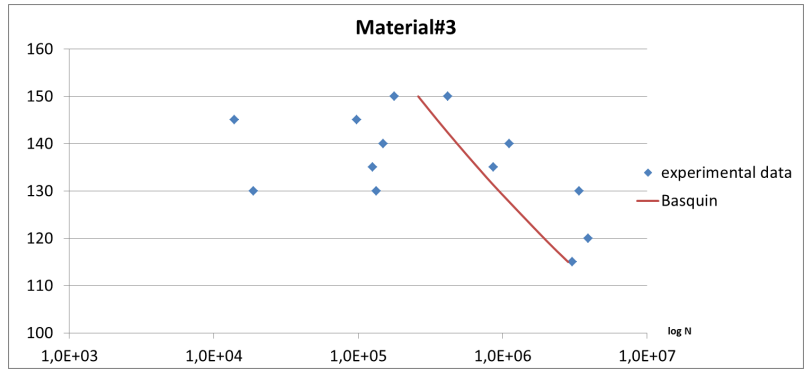

Figure 6. Optimal fatigue model for Material\#3.

\subsection{Reliability Analysis in JMP}

In JMP custom tables can be created, or files of different formats (Excel, SAS, text files, ...) can be processed. For a correct data input, the appropriate settings in the import wizard must be applied.

In order to determine the best distribution for the measured values, a life distribution is performed 11 . The program returns a table with the appropriate distributions for the respective materials. JMP sorts them in descending order, the best model being on the top. The distribution of the measured data is weighted by 3 criteria.The Akaike Information Criterion (AIC), the Bayesian Information Criterion (BIC) and Loglikelihood (maximum probability) used as estimation methods for the selection of models in statistics. 
Tables 1. 2 and 3 show the "Model Comparisons" for each materials. According to the distribution analysis in JMP, the measured values from Material\#1 follows a natural logarithmic distribution, from Material\#2 a Frechet distribution and from Material\#3 a Weibull distribution.

\begin{tabular}{|c|c|c|c|}
\hline Distribution & AICc & Loglike & BIC \\
Lognormal & 503.65435 & 498.90435 & 504.79323 \\
Weibull & 503.66928 & 498.91928 & 504.80816 \\
Frechet & 505.47261 & 500.72261 & 506.61149 \\
Loglogistic & 505.74393 & 500.99393 & 506.88280 \\
Exponential & 518.31862 & 516.08332 & 519.02776 \\
LEV & 538.93104 & 534.18104 & 540.06992 \\
Logistic & 546.20378 & 541.45378 & 547.34266 \\
Normal & 550.30607 & 545.55607 & 551.44495 \\
SEV & 563.21318 & 558.46318 & 564.35205 \\
\hline
\end{tabular}

TABle 1. Comparisons of the distribution of the measured data for Material\#1.

\begin{tabular}{|c|c|c|c|}
\hline Distribution & AICc & Loglike & BIC \\
Frechet & 297.91571 & 292.41571 & 297.21150 \\
Lognormal & 299.16181 & 293.66181 & 298.45760 \\
Loglogistic & 300.06766 & 294.56766 & 299.36345 \\
Weibull & 301.43592 & 295.93592 & 300.73171 \\
Exponential & 305.28286 & 302.83841 & 305.23631 \\
LEV & 320.31613 & 314.81613 & 319.61192 \\
Logistic & 326.13477 & 320.63477 & 325.43056 \\
Normal & 329.93763 & 324.43763 & 329.23342 \\
SEV & 337.33938 & 331.83938 & 336.63517 \\
\hline
\end{tabular}

TABle 2. Comparisons of the distribution of the measured data for Material\#2.

\begin{tabular}{|c|c|c|c|}
\hline Distribution & AICc & Loglike & BIC \\
Weibull & 407.25467 & 402.16376 & 407.44188 \\
Loglogistic & 409.33460 & 404.24369 & 409.52181 \\
Lognormal & 409.74094 & 404.65003 & 409.92814 \\
Frechet & 415.33191 & 410.24100 & 415.51912 \\
Exponential & 416.03305 & 413.69971 & 416.33877 \\
LEV & 432.98688 & 427.89597 & 433.17409 \\
Logistic & 439.48273 & 434.39182 & 439.66994 \\
Normal & 439.98404 & 434.89313 & 440.17125 \\
SEV & 445.68650 & 440.59560 & 445.87371 \\
\hline
\end{tabular}

TABle 3. Comparisons of the distribution of the measured data for Material\#3.

Based on this findings, the durability evaluation was performed. Tables 4, 5 and 6 display the mean estimates for each material. $\sigma$ is the standard deviation from the measured data, $\beta_{0}$ and $\beta_{1}$ denote positional and shape parameters. StdError stands for standard error and describes the standard deviation, but from the estimate function. Additionally the tables show for each estimation the $95 \%$ confidence interval.

$\mu$ is the estimated average value of cycles, which is dependent on the loading. Strictly speaking, this designation should be $\log \mu$, because the relations are from logarithmic nature.

\begin{tabular}{|c|c|c|c|c|}
\hline Par. & Estimate & StdError & Low95\% & Up95\% \\
$\beta_{0}$ & 79.25810 & 6.98804 & 64.83945 & 93.67675 \\
$\beta_{1}$ & -14.17640 & 1.44835 & -17.16484 & -11.18796 \\
$\sigma$ & 0.92287 & 0.14971 & 0.69226 & 1.31688 \\
\hline
\end{tabular}

TABLE 4. Mean estimations for Material\#1.

$$
\mu=79.2581-14.1764 \cdot \log (\text { loading })
$$

\begin{tabular}{|c|c|c|c|c|}
\hline Par. & Estimate & StdError & Low95\% & Up95\% \\
$\beta_{0}$ & 126.9802 & 20.30212 & 82.06733 & 168.4553 \\
$\beta_{1}$ & -22.7520 & 3.98476 & -30.90587 & -13.9531 \\
$\sigma$ & 0.6862 & 0.15914 & 0.45366 & 1.1457 \\
\hline
\end{tabular}

TABLE 5. Mean estimations for Material\#2.

$$
\mu=126.9802-22.75204 \cdot \log (\text { loading })
$$

\begin{tabular}{|c|c|c|c|c|}
\hline Par. & Estimate & StdError & Low95\% & Up95\% \\
$\beta_{0}$ & 86.41222 & 14.55656 & 53.28540 & 112.4174 \\
$\beta_{1}$ & -14.89503 & 2.94585 & -20.13681 & -8.1682 \\
$\sigma$ & 1.18943 & 0.25506 & 0.80843 & 1.8933 \\
\hline
\end{tabular}

TABLE 6. Mean estimations for Material\#3.

$$
\mu=86.41222-14.89503 \cdot \log (\text { loading })
$$

Figures 7] 8 and 9 illustrate the quantile analysis. The quantile is a measure of location in statistics. It represents a threshold. A certain amount of the value is below, the residual amount above this threshold. Using the example of Material\#1 the threshold is 21675.91 cycles. That means here that for a loading of 132.5 there is a $50 \%$ probability of failure. The blue dotted lines represent the $95 \%$ confidence interval.

JMP can also perform a custom estimate. For example, the failure probability at the endurance limit is calculated for each material. The results are given in figures 10. 11 and 12 In the case of Material\#3 the material can sustain $4.7^{*} 10^{6}$ cycles at a loading of 93 and a failure probability of $5 \%$. At a loading of 93 and cycles of $40^{6}$ the failure probability becomes $4.4 \%$.

\section{Conclusion}

Due to the very large scatter of the measurement results, the determination of the most suitable model 


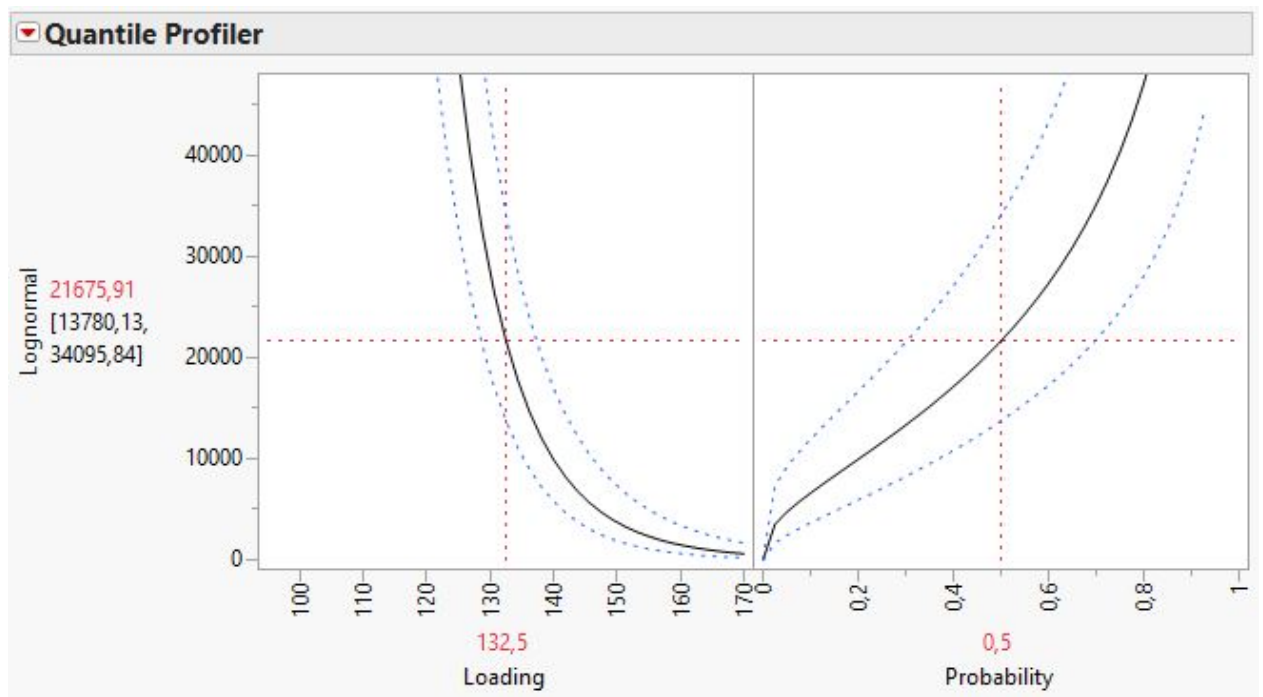

FiguRE 7. Quantile analysis for Material\#1.

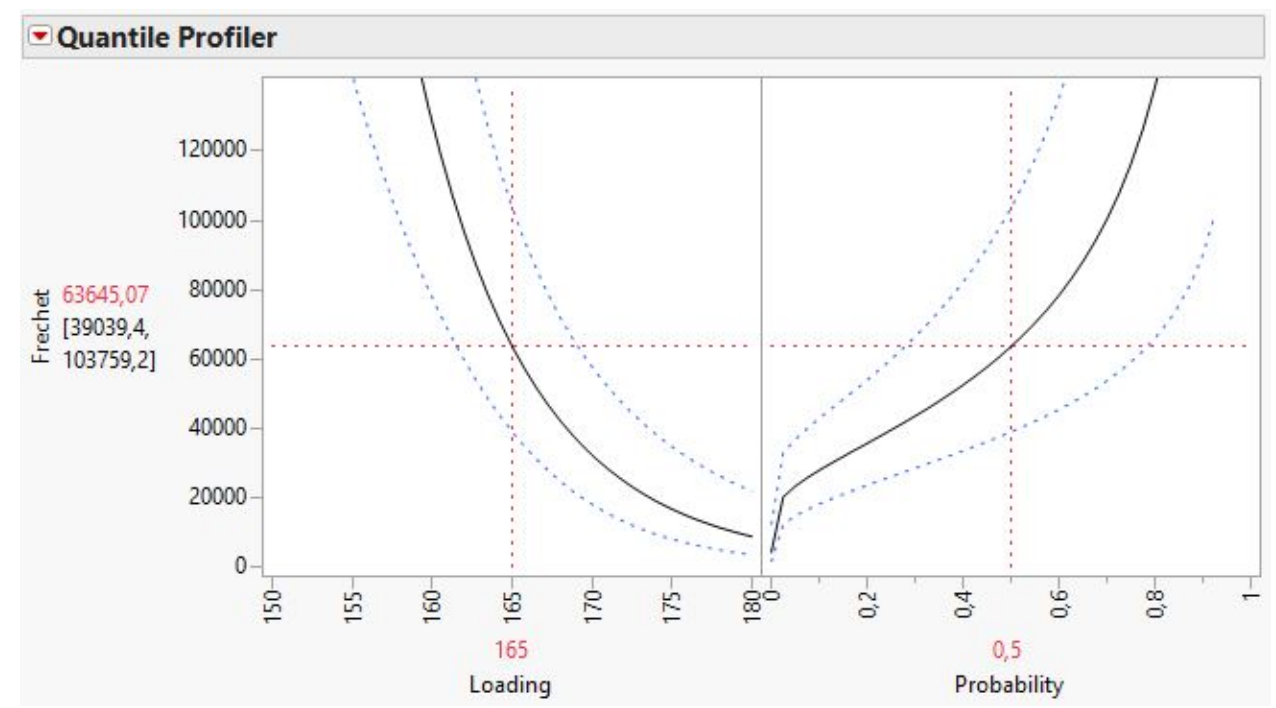

FiguRE 8. Quantile analysis for Material\#2.

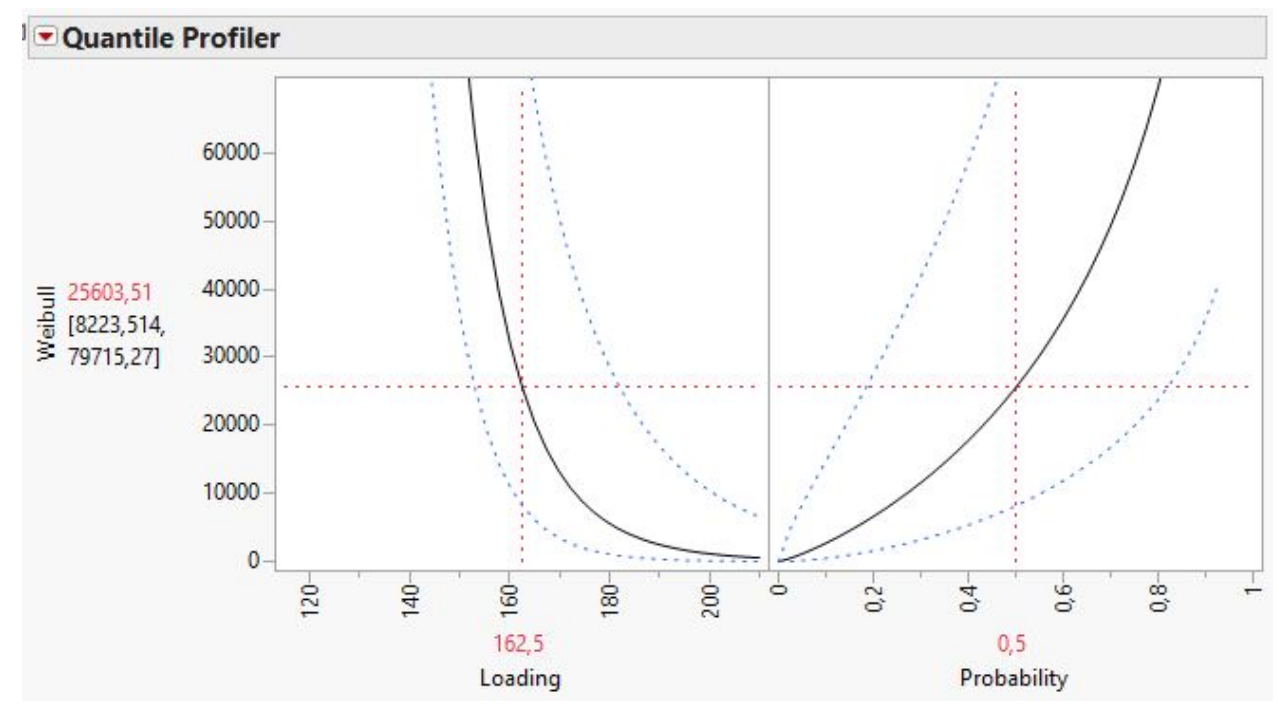

Figure 9. Quantile analysis for Material\#3. 


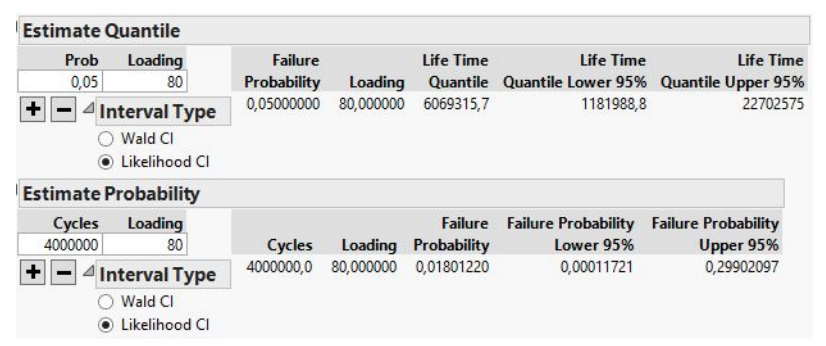

Figure 10. Custom estimate for Material\#1.

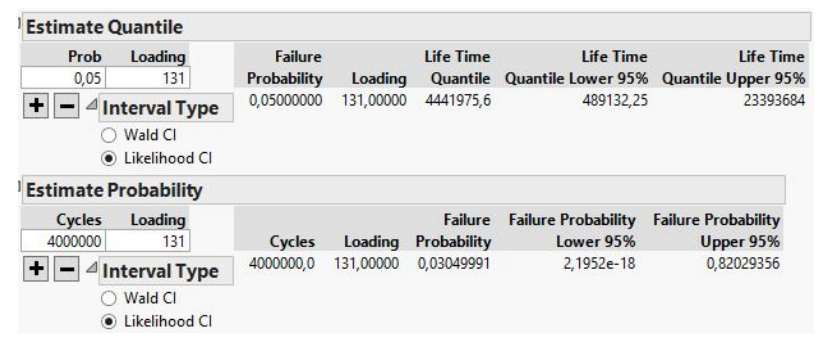

Figure 11. Custom estimate for Material\#2.

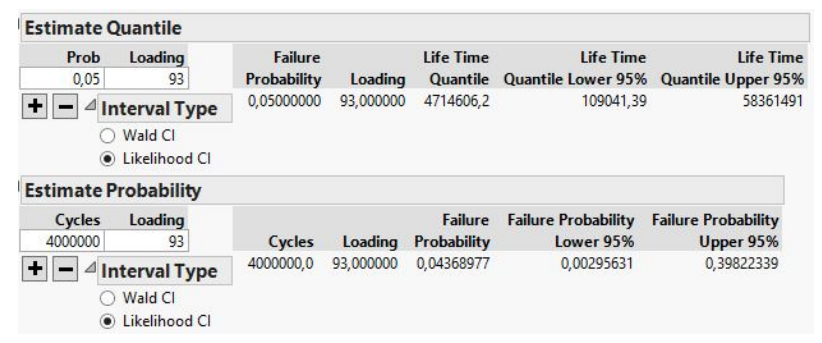

Figure 12. Custom estimate for Material\#3.

to fit the experimental data was a major challenge. In some cases, one gets very unsatisfactory graphical representations of the models. By the application of several fatigue models, at least one suitable model for each material could be found. By the implementation of statistical analysis also the large outliers could be included in the parameter estimation.

JMP is a powerful tool for statistical analysis. Especially, the analysis of the failure probability is a very important feature. The estimate in JMP within the experimental data range leads to slightly different prediction than that of the fatigue models. Extrapolation with JMP out of range is highly dependent on the quality of the measurement data and therefore, does not always leads to plausible results.

\section{LIST OF SYMBOLS}

$A, B, C, D$ material parameter

$n$ number of cycles

$\Delta \sigma$ loading amplitude

$\Delta \sigma_{\mathrm{o}}$ loading amplitude at endurance limit

$\Delta \sigma_{s t}$ loading amplitude at ultimate strength

$\sigma$ standard deviation

$\beta_{\mathrm{o}}$ positional parameter in JMP

$\beta_{1}$ shape parameter in JMP

$\mu$ estimation for the mean value

\section{REFERENCES}

[1] T. K. Anastasios P. Vassilopoulos. Fatigue of Fiber-reinforced Composites. Springer Verlag London Limited, London, 2011.

[2] M. V. Dieter Radaj. Ermüdung - Grundlagen für Ingenieure. Springer Verlag Berlin, Heidelberg, 1995, 2003, 2007.

[3] A. F.-C. E. Castillo. A Unified Statistical Methodology for Modeling Fatigue Damage. Springer Science + Business Media B. V., 2009.

[4] U. Heidelberg. http://www.physi.uni-heidelberg.de/Einrichtungen/ AP/Elearning/index.php/animationen/37-anpassungvon-funktionen-an-messdaten/52-die-methode-derkleinsten-fehlerquadrate 2013. Universität Heidelberg, 2013.

[5] S. J. M. C. R. A. Schneider. Best Practice Guide on Statistical Analysis of Fatigue Data. TWI Cambridge, UK, 2003.

[6] A. S. for Testing, Materials. Statistical Analysis of Fatigue Data. ASTM, 1981.

[7] S. J. Miller. The Method of Least Squares. Brown University, Providence, RI 02912, US.

[8] T. E. J. S. Udo Kuckartz, Stefan Rädiker. Statistik Eine verständliche Einfürung. Springer Fachmedien Wiesbaden GmbH, 2010.

[9] A. F. Hans Diefenbacher. Statistik - Eine verständliche Einfürung. Andreas Frank \& Ventus Publishing ApS, 2006.

[10] P. Schmolck. Methoden der Reliabilitätschätzung. Universität der Bundeswehr München, 2007.

[11] S. I. Inc. JMP Version 11 Documentation. SAS Institute Inc., 2013. 